\title{
SIMULATING SMOKE MOVEMENT THROUGH LONG VERTICAL SHAFTS IN ZONE-TYPE COMPARTMENT FIRE MODELS
}

\section{Leonard Y. Cooper}

Building and Fire Research Laboratory

Gaithersburg, Maryland 20899

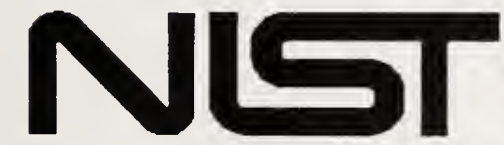

United States Department of Commerce

$$
\text { ogy Administration }
$$

$Q C$

II Institute of Standards and Technology

100

.456

NO. 5526 



\section{SIMULATING SMOKE MOVEMENT THROUGH LONG VERTICAL SHAFTS IN ZONE-TYPE COMPARTMENT FIRE MODELS}

Leonard Y. Cooper

November 1994

Building and Fire Research Laboratory

National Institute of Standards and Technology

Gaithersburg, MD 20899

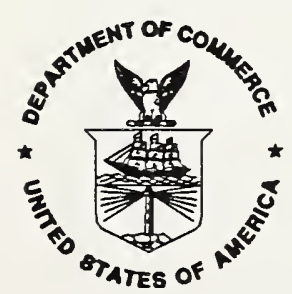

U.S. Department of Commerce

Ronald H. Brown, Secretary

Technology Administration

Mary L. Good, Under Secretary for Technology

National Institute of Standards and Technology

Arati Prabhakar, Director 

TABLE OF CONTENTS $\ldots \ldots \ldots \ldots \ldots \ldots \ldots \ldots \ldots \ldots \ldots \ldots \ldots \ldots \ldots \ldots \ldots$

LIST OF FIGURES $\ldots \ldots \ldots \ldots \ldots \ldots \ldots \ldots \ldots \ldots \ldots \ldots \ldots \ldots \ldots \ldots \ldots \ldots \ldots \ldots \ldots$ iv

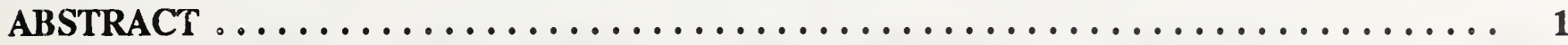

INTRODUCTION ..................................... 1

Zone-Type Compartment Fire Modeling Concepts ................. 1

A Limitation of the Two-Layer Quasi-Steady-Buoyant-Plume Modeling Approach ... 2

A Strategy for Modeling Flow Through Shaft-Like Spaces in Zone-Type Fire Compartment Models; Objective of This Work ............... 2

COMBINED BUOYANCY- AND VENTILATION-DRIVEN FLOW OF A PERFECT GAS THROUGH A LONG VERTICAL SHAFT $\ldots \ldots \ldots \ldots \ldots \ldots \ldots \ldots \ldots, \ldots$

TURBULENT FLUCTUATIONS IN THE SHAFT FLOW $\ldots \ldots \ldots \ldots \ldots \ldots \ldots, \ldots$

The Turbulence Equations .......................... 4

Experiments in Shafts With Unstable Density Configurations; A Qualitative

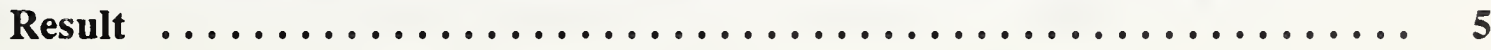

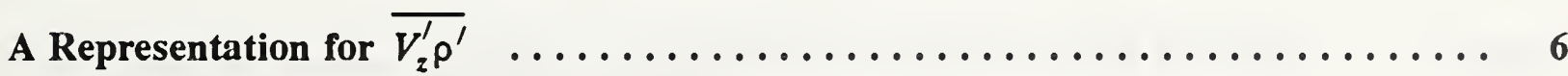

UNSTEADY EXPERIMENTS TO VERIFY EQS. (9)-(11) AND THE VALUE OF $K$. $\ldots \ldots \quad 7$ Description of the Two Phase Experiments $\ldots \ldots \ldots \ldots \ldots \ldots \ldots \ldots \ldots, 7$ The Initial Value Problem for the Diffusion Front: Phase I $\ldots \ldots \ldots \ldots \ldots, 8$ Relaxation of the Diffusion Front: Phase II ................... 11

SUMMARY, CONCLUSIONS, AND RECOMMENDATIONS $\ldots \ldots \ldots \ldots \ldots \ldots \ldots, 12$

REFERENCES $\ldots \ldots \ldots \ldots \ldots \ldots \ldots \ldots \ldots \ldots \ldots \ldots \ldots \ldots \ldots \ldots \ldots \ldots \ldots$

NOMENCLATURE $\ldots \ldots \ldots \ldots \ldots \ldots \ldots \ldots \ldots \ldots \ldots \ldots \ldots \ldots \ldots \ldots \ldots \ldots$ 


\section{LIST OF FIGURES}

Page

Figure 1. Sketch of a facility where traditional, two-layer, zone-type, modeling concepts are not uniformly applicable. ........................ 17

Figure 2. Sketch of the simulated experiments of Cannon and Zukoski [1] . . . . . . 18

Figure 3. Position of the diffusion front in Phase I: small- $\epsilon$ solution of Eq. (22) for different values of $K$; and values measured in the salt-water/fresh-water experiments of Cannon and Zukoski [1].................. 19

Figure 4. Phase I solutions of Eqs. (23), including moderate-to-large $\epsilon$ : (a) $\theta(\eta ; \epsilon)$; (b) $\Gamma(\epsilon) ;\left(\right.$ c) $\eta_{F R O N T}(\epsilon)$; and (d) $\psi\left[\eta_{F R O N T}(\epsilon) ; \epsilon\right] . \ldots \ldots \ldots \ldots \ldots$

Figure 5. Plot of $Q(\epsilon)$ of Eq. (29) from Phase II solutions of Eqs. (27) and (28). . . . 24

Figure 6. Plots for Phase II: Eqs. (28)-(29) at $z=L$ for $\epsilon=0$; salt-water/fresh-water data of Cannon and Zukoski [1] using $K=0.56$ and least-squares-fit value,

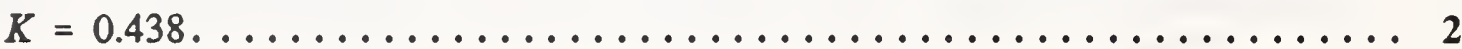

Figure 7. Plots for Phase II: Eqs. (28)-(29) at $z=L$ for $\epsilon=0.22,0.38$, and 4.0; heavy-gas/light-gas data of Cannon and Zukoski [1] for $\epsilon=0.22,0.38$, and 4.0

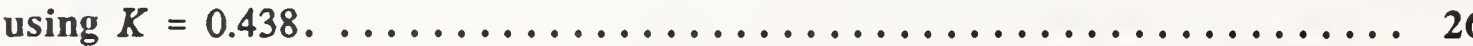




\title{
SIMULATING SMOKE MOVEMENT THROUGH LONG VERTICAL SHAFTS IN ZONE-TYPE COMPARTMENT FIRE MODELS
}

\begin{abstract}
A limitation of traditional zone-type compartment fire modeling concepts is identified; namely, the inadequacy of two-layer quasi-steady-buoyant-plume analyses to simulate the fire-generated environment in room configurations with large height-to-span ratios, e.g., elevator shafts and long, vertical, ventilation shafts and ducts. A possible means of removing this limitation is developed. This involves a method of analysis and associated model equations that can be implemented and used to advance zone-type models. The model equations simulate time-dependent flows in a long, ventilated, vertical shaft/duct with an arbitrary vertical density distribution, including one or more intervals along the shaft/duct length where the vertical distribution of the average cross-section density may be unstably stratified, i.e., density increasing with increasing elevation. The model equations are partially verified by favorable comparisons between solutions and previously published data from unsteady experiments in long vertical tubes involving initially unstable configurations: salt-water over freshwater and heavy-gas over light-gas. Additional verification of the proposed equation set with cold-air over hot-air systems and with fire-driven smoke flows, both of which involve gas-to-surface heat transfer, is required before this model can be used with confidence in professional practice.
\end{abstract}

\section{INTRODUCTION}

\section{Zone-Type Compartment Fire Modeling Concepts}

Zone-type computer fire models are important practical tools for simulating mathematically the dynamics of fire and smoke spread through multi-room facilities. In such models, rooms are assumed to be connected to one another or to the outside environment by vents (i.e., holes) in bounding partitions. Room-to-room cross-vent exchanges of fresh air and/or smoke are determined from momentum considerations (e.g., the Bernoulli equation); these exchanges being driven by room-toroom, cross-vent, hydrostatic pressure-differences. The vertical hydrostatic pressure distribution in any particular room of a modeled facility is calculated from the time-dependent pressure at the floor (or at some other fixed elevation in the room) and from the floor-to-ceiling distribution of density. Time-dependent pressure at the reference elevation and density distribution in each room of the facility re included as variables computed by the fire model. Variations in pressures and flows throughout the facility are determined from the buoyancy of fire-generated layers of smoke that grow in thickness and temperature at the upper portions of one or more rooms of the facility, by nonuniform, wind-generated, stagnation pressure distributions that surround the ("leaky") external surfaces of the facility, and/or by stack effects (elevation-dependent pressure differences between the inside and outside of a tall facility generated by differences of outside density/temperature and climate-controlled inside density/temperatures. Some fire models also have a capability for simulating the effect on pressures and flows of operating ventilation (HVAC) system components. In the latter case, the current state of zone-type fire modeling technology is such that duct pressure distributions and duct flow and mixing are generally calculated or specified without a full accounting of the buoyancy of the flowing gas.

The fire-generated environment in any particular room of a facility is generally approximated by two stably stratified layers; an upper, elevated-temperature, and relatively-buoyant smoke layer and a cooler and less-buoyant lower layer. Each layer is assumed to have spatially-uniform time-dependent 
properties. Prior to the time when smoke is introduced to a room, either from a fire contained in the room or by smoke inflow through a vent or ventilation duct inlet, the room would be filled with an ambient-temperature lower layer, i.e., the interface separating the upper layer from the lower layer would be at its initial, ceiling elevation.

When a source of buoyant smoke is introduced to a room below the smoke layer it rises as a buoyant plume toward the smoke layer interface, entraining laterally lower-layer gases during its ascent. In the current state of technology of zone-type models, the flow dynamics above a source of buoyant smoke is modeled mathematically as a purely-buoyant quasi-steady plume in a large quiescent environment. Here, "large" is intended to indicate that the volume of the lower layer is large compared to the volume containing the significant plume-flow activity. Also, implicit in the uniform layer approximation is the assumption that, when a plume enters a layer, the characteristic time of mixing of the penetrating plume gases with the bulk of the receiving layer is small compared to characteristic times of the overall phenomena being simulated.

\section{A Limitation of the Two-Layer Quasi-Steady-Buoyant-Plume Modeling Approach}

The modeling strategy which uses the concepts of one-to-two uniform layers per room, room-to-room mass exchanges by vent flows, and layer-to-layer mass exchange by quasi-steady buoyant plumes has proven to be very robust. However, there are important practical room configurations and associated fire scenarios where these basic concepts are inadequate. If the basic concepts are not applicable to a particular room configuration, then, to the extent that room plays a significant role in the spread of fire and smoke throughout the facility (e.g., the room is the connecting flow path between the room of fire origin and a threatened space), the inadequacy of the simulation in that room can render inadequate the entire simulation.

Let $L$ and $d$, be the characteristic height and horizontal span, respectively, of a room configuration. Then, in view of the above discussion, for rooms with $L / d \gg 1$ (shafts or ducts) it is not reasonable to expect a traditional, two-layer, zone-type, fire modeling approach to lead to a successful simulation of fire environments if such spaces are described and treated in the manner of a standard, two-layer, room element, with plumes, etc. For such room configurations, some of the previously mentioned basic modeling assumptions become invalid. Thus, 1) as the plume rises and spreads, its volume eventually becomes significant and it starts to fill a large fraction of the section of the shaft/duct, 2) it is not reasonable to expect that characteristic times of mixing in the shaft will generally be small compared to characteristic times of interest, and 3) there is no basis for support for a uniform, twolayer approximation to the density/temperature distribution. An illustration of a generic problematic facility and room configuration is presented in Figure 1. In the facility shown, traditional modeling concepts would typically be applicable in the small- $L / d$ room of fire origin, the upper floors, etc., but they would not be applicable in the large- $L / d$ shaft.

\section{A Strategy for Modeling Flow Through Shaft-Like Spaces in Zone-Type Compartment Fire Models; Objective of This Work}

For the traditional two-layer, etc., zone-type modeling approach to be valid, large- $L / d$ spaces in a multi-room facility need to be treated as a special class of room configuration. Fire-generated environments there need to be simulated with a method of analysis that 1) uses a valid intra-room fire dynamics modeling approach and 2) can be implemented in the compartment fire model to study 
fire scenarios in facilities which include other "standard," i.e., small-to-moderate- $L / d$, room elements. The purpose of this paper is to develop a framework for such an analysis.

Once a method of analyzing large- $L / d$ spaces is developed and verified, it could be incorporated into an existing multi-room fire model. The revised model would then be capable of describing smoke movement through shaft-like spaces that may be included in the design of a particular facility of interest. The strategy for carrying out simulations with the revised fire model would involve categorizing each room of a modeled facility as either a small-to-moderate- $L / d$ space or a large- $L / d$ space, where results of future research are expected to lead to appropriate rules for such categorization. Then, when carrying out a simulation, the fire model would invoke the traditional, two-layer, isolated-plume, etc. model equations for the small-to-moderate- $L / d$ spaces and the new model equations for large- $L / d$ spaces.

Note that an even more advanced strategy might consider an additional, third class of facility space, designated as a "moderate- $L / d$ " space. The analysis of the fire environment in the "moderate- $L / d$ " space would be designed to bridge the gap between small- and large- $L / d$ spaces by including important modeling features of both.

\section{COMBINED BUOYANCY- AND VENTILATION-DRIVEN FLOW OF A PERFECT GAS THROUGH A LONG VERTICAL SHAFT}

Consider flow of a perfect gas through a vertical shaft of length $L$. Let the section of the shaft have a characteristic dimension, $d$, and assume that the shaft is long in the sense that $L / d>1 . \rho$ and $T$, the density and temperature of the gas averaged across the section, vary along the length of the shaft. In general, the shaft is ventilated; inflow or outflow of the gas can occur at vents, in the walls of the shaft, or at its ends. Inflowing gas has specified density and temperature, $\rho_{V E N T}$ and $T_{\text {VENT }}$, respectively, which can vary along the shaft. Outflowing gas is assumed to be at density and temperature, $\rho$ and $T$, respectively. The local rate of mass addition due to ventilation is $\dot{m}_{V E N T}^{\prime \prime \prime}$ (rate of mass added to the shaft per unit volume). Heat is transferred between the gas and the shaft surface at the rate $\dot{q}_{H T}^{\prime \prime \prime}$ (rate of heat transferred to the gas in the shaft per unit volume).

Assume that the only possible significant component of flow velocity is along the axis of the shaft in the vertical $\mathrm{z}$ direction and let its average across a section be $V_{z}$. Then, conservation of mass and energy, and the equation of state leads to: 


$$
\begin{gathered}
\partial \rho / \partial t+\partial\left(V_{z} \rho\right) / \partial z=\dot{m}_{V E N T}^{\prime \prime \prime} \\
\rho C_{p}\left(\partial T / \partial t+V_{z} \partial T / \partial z\right)=-\partial(k \partial T / \partial z) / \partial z+C_{p}\left(T_{V E N T}-T\right) \dot{m}_{V E N T}^{\prime \prime \prime}+\dot{q}_{H T}^{\prime \prime \prime} \\
\rho T=\rho_{A M B} T_{A M B}=\text { constant }
\end{gathered}
$$

where $C_{p}$ is the specific heat at constant pressure, $k$ is the thermal conductivity, and $\rho_{A M B}$ and $T_{A M B}$ are the density and temperature at some ambient reference state.

Note that the formulation of Eq. (3) uses the uniformly valid assumption that variations in pressure throughout the length of the shaft are negligible compared to the characteristic pressure of the ambient reference state. At a point in the analysis where momentum considerations are required, for example in the computation of pressure differences across vents for the purpose of estimating vent flow rates, estimates of the relatively-small shaft pressure variations (e.g., the contribution of hydrostatic pressure variation) would be important and they must be estimated properly.

Using Eqs. (1) and (3) in Eq. (2) leads to the following simplified alternative to Eq. (2):

$$
\partial V_{z} / \partial z=\left[-\partial(k \partial T / \partial z) / \partial z+C_{p} T_{V E N T} \dot{m}_{V E N T}^{\prime \prime \prime}+\dot{q}_{H T}^{\prime \prime \prime}\right] /\left(\rho_{A M B} T_{A M B} C_{p}\right)
$$

\section{TURBULENT FLUCTUATIONS IN THE SHAFT FLOW}

\section{The Turbulence Equations}

Eqs. (1), (3), and (4) are now revised to account for the effect of possible turbulent fluctuations in the shaft flow. Time-averaged ("barred") and fluctuating ("primed") components of the variables are introduced

$$
\rho=\bar{\rho}+\rho^{\prime} ; \quad T=\bar{T}+T^{\prime} ; \quad V_{z}=\bar{V}_{z}+V_{z}^{\prime}
$$

Reynolds averaging, neglect of the generally insignificant effect of heat conduction through the gas, along the length of the shaft, as derived from the time-averaged temperature distribution, and the assumption that time-averaged values of $\rho$ and $T$ satisfy the equation of state lead to the following modified equation set: 


$$
\begin{gathered}
\partial \bar{\rho} / \partial t+\bar{V}_{z} \partial \bar{\rho} / \partial z+\partial\left(\overline{V_{z}^{\prime} \rho}\right) / \partial z=\dot{m}_{V E N T}^{\prime \prime \prime}\left(1-\bar{\rho} / \rho_{V E N T}\right)-\left(\bar{\rho} / \rho_{A M B}\right) \dot{q}_{H T}^{\prime \prime \prime} /\left(T_{A M B} C_{p}\right) \\
\partial \overline{V_{z}} / \partial z=\left(C_{p} T_{V E N T} \dot{m}_{V E N T}^{\prime \prime \prime}+\dot{q}_{H T}^{\prime \prime \prime}\right) /\left(\rho_{A M B} T_{A M B} C_{p}\right) \\
\bar{\rho} \bar{T}=\rho_{A M B} T_{A M B}=\text { constant }
\end{gathered}
$$

Turbulent fluctuations leading to significant values of the term $\overline{V_{z}^{\prime} \rho^{\prime}}$ in Eq. (6) will occur along the length of a vertical shaft where there are buoyancy-generated instabilities in the vertical density distribution, i.e., where, locally, the density is increasing with elevation, $\partial \bar{\rho} / \partial z>0$. Indeed, such instabilities are the driving force for the turbulent-like mixing phenomenon which is of particular interest here. Also, shaft flow scenarios of present interest are such that $\bar{V}_{z}$ is small enough as never to lead to turbulent fluctuation enhancements that would significantly affect the value of $\overline{V_{z}^{\prime} \rho^{\prime}}$. For example, at elevations along the shaft where the gas is stably stratified, i.e., where $\partial \bar{\rho} / \partial z \leq 0$, there will be no buoyancy-driven turbulence and it is reasonable to assume that $\partial\left(\overline{V_{z}^{\prime} \rho}\right) / \partial z$ in Eq. (6) can be neglected completely.

Solutions to Eqs. (6)-(8) with appropriate initial and boundary conditions would generally provide a description of the fire environment that develops in ventilated, shaft-like, room configurations. However, to actually implement this equation set it is necessary to develop supplementary model equations to represent $\overline{V_{z}^{\prime} \rho^{\prime}}$ of Eq. (6) and the source terms, $\dot{m}_{V E N T}^{\prime \prime \prime}$ and $\dot{q}_{H T}^{\prime \prime \prime}$, of Eqs. (6) and (7). The focus of the remainder of the present work is on obtaining a general representation for $\overline{V_{z}^{\prime} \rho^{\prime}}$.

\section{Experiments in Shafts With Unstable Density Configurations; A Qualitative Result}

During experiments in vertical tubes of diameter d, involving initially unstable configurations of saltwater over fresh-water and prior to any end effects, Cannon and Zukoski [1] have observed fluid mixing by buoyancy-generated instabilities. This involved overturning and fluctuating eddies of characteristic dimension $d$. Also, as observed in similar unstable systems by Cannon and Zukoski [1] (heavy gas over light gas), Baird and Rice [2] (rising bubble columns), and Gardner [3] (saltwater/fresh-water systems and experiments on water rising through more dense carbon tetrachloride), the propagation of buoyancy-driven turbulent fluctuations through the buoyantly unstable column behaves phenomenologically as a diffusion-like process. This is represented by the $\partial\left(\overline{V_{z}^{\prime} \rho}\right) / \partial z$ term in Eq. (6). 


\section{A Representation for $\overline{V_{z}^{\prime} \rho^{\prime}}$}

Shaft flow systems involving negligible $\bar{V}_{z}$. Compressibility was negligible in all the above-referenced experiments. Therefore, $\bar{V}_{z}$ was negligible everywhere, and it would appear that insights on a practical and general representation for $\overline{V_{z}^{\prime} \rho^{\prime}}$ in Eq. (6) can be obtained from the results of these relatively-simple negligible- $\bar{V}_{z}$ systems.

Consider shaft flow systems of Eqs. (6)-(8) with negligible $\bar{V}_{z}$ and negligible mass addition or heat transfer along the length of the shaft. For such systems, Eq. (6) becomes

$$
\left.\partial \bar{\rho} / \partial t+\partial \overline{\left(V_{z}^{\prime} \rho\right.}\right) / \partial z=0
$$

Defining $D$ as the eddy diffusivity for buoyancy-driven turbulence, Eq. (9), with

$$
\overline{V_{z}^{\prime} \rho^{\prime}}=-D \partial \bar{\rho} / \partial z
$$

has been used in each of the above-referenced systems. It is also used by Cannon and Zukoski [1] in the modeling of a perfect gas air system, i.e., low temperature over high-temperature air, with nonzero $\dot{q}_{H T}^{\prime \prime \prime}$. In Eq. (10), $D$ is expected to be an increasing function of $\partial \bar{\rho} / \partial z$ for $\partial \bar{\rho} / \partial z>0$, and to be zero for $\partial \bar{\rho} / \partial z \leq 0$ (i.e., buoyancy-driven turbulent diffusion is suppressed at elevations where the fluid column is stably stratified).

Cannon and Zukoski [1] expect $D$ to be proportional to $w^{\prime}$, a characteristic velocity of the turbulent fluctuations, and to satisfy

$$
D \sim w^{\prime} l=[(g / \bar{\rho}) \partial \bar{\rho} / \partial z]^{1 / 2} l^{2}
$$

where $l$ is a characteristic length of the fluid mixing process. Here, $l$ is taken to be the characteristic dimension of the observed buoyancy-driven eddies, namely, $d$.

$$
D=\left\{\begin{array}{l}
K d^{2}[(g / \bar{\rho}) \partial \bar{\rho} / \partial z]^{1 / 2} \text { if } \partial \bar{\rho} / \partial z>0 \\
0 \text { if } \partial \bar{\rho} / \partial z \leq 0
\end{array}\right.
$$


where $K$ is a constant and where the form of Eq. (11) is generally equivalent to that presented by Baird and Rice [2] and Gardner [3].

A determination of $K$; a steady-state experiment. To determine $K$, Gardner [3] considered the particular problem of quasi-steady turbulent diffusion in a long shaft connecting two relatively large vessels of incompressible fluids, with density of the fluid in the top and bottom vessels, $\rho_{\text {TOP }}$ and $\rho_{B O T}<\rho_{\text {TOP }}$, respectively; where $\Delta \rho=\rho_{\text {TOP }}-\rho_{B O T}$ is such that $\Delta \rho /\left(\rho_{T O P}+\rho_{B O T}\right)<<1$. To insure that $V_{z}=0$, at least one of the vessels is fully enclosed. This problem was studied experimentally by Mercer and Thompson [4] with salt-water/freshwater systems and circular shafts of diameter, $d$.

For the above class of problem, $\bar{\rho} \approx \rho_{A V E}=\left(\rho_{T O P}+\rho_{B O T}\right) / 2$ and

$$
D \approx K d^{2}\left[\left(g \Delta \rho / \rho_{A V E}\right) d c_{T O P} / d z\right]^{1 / 2} ; \quad-D d c_{B O T} / d z=D d c_{T O P} / d z \approx D / L=4 Q /\left(\pi d^{2}\right)
$$

where $Q$ is the volume flow rate of the fluid in the lower vessel diffusing up through the shaft and into the top vessel and where $c_{\text {TOP }}, c_{B O T}$ are the concentrations in the shaft of the top and bottom fluids, respectively.

With quasi-steady systems of salt water over fresh water, Mercer and Thompson [4] determined $Q$ of fresh water from the lower vessel by measuring salt concentrations and the rates of mass decrease of the salt water in a top vessel. Using these results in Eqs. (12), Gardner [3] determined

$$
K=K_{G}=0.56
$$

In contrast to this, Baird and Rice [2] analyzed the literature on rising bubble columns and related systems where gas flow rates are large enough for the bubble wakes to interact strongly and to generate turbulence in the opposing fluid motion. For such systems they determined the "order-ofmagnitude estimate"

$$
K=K_{B R}=0.21
$$

\section{UNSTEADY EXPERIMENTS TO VERIFY EQS. (9)-(11) AND THE VALUE OF $K$}

\section{Description of the Two Phase Experiments}

The unsteady experiments of Cannon and Zukoski [1] included the configuration of Figure 2 involving a tube of length $L$, closed at the upper end, $z=L$, and inserted into a relatively large 
vessel of density $\rho_{B O T}$. The tube was initially filled with fluid of density $\rho_{\text {TOP }}>\rho_{\text {BOT }}$. The experiments were initiated by allowing the fluid in the tube to mix with the fluid in the lower vessel. The mixing phenomena in the tube involves two phases, Phases I and II. Phase I begins when the experiment is initiated. A diffusion front at elevation $z=z_{F R O N T}(t), z_{F R O N T}(t=0)=0$, rises in the tube, and in $0 \leq z \leq z_{F R O N T}(t)$ there is mixing due to non-zero $\partial \bar{\rho} / \partial z$. Phase I is completed and Phase II is initiated at time $t=t_{A R R V A L}$, when $z_{\text {FRONT }}=L$, i.e., at the time of arrival at the top of the tube of the diffusion front, when the effects of the diffusive mixing begin to modify significantly the density at the upper end of the tube. During the remainder of Phase II, for $t>t_{\text {FRONT }}$, the unstable density distribution drives turbulent-like mixing eddies through the length of the tube. The distribution eventually relaxes to the uniform state, $\rho=\rho_{\text {BOT }}$.

Data from both Phases I and II of the Cannon and Zukoski experiments [1] can be used to verify the model Eqs. (9)-(11) and the value of $K$. The next section addresses the Phase I phenomena.

The Initial Value Problem for the Diffusion Front: Phase I

Problem Formulation and Similarity. From Eqs. (9)-(11), the initial value problem for Phase I of the above-referenced experiments is

$$
\begin{gathered}
\frac{\partial \theta}{\partial \tau}=\frac{\partial}{\partial \zeta}\left[\frac{1}{(1+\epsilon \theta)^{1 / 2}}\left(\frac{\partial \theta}{\partial \zeta}\right)^{3 / 2}\right] \\
\theta(\zeta=0, \tau>0 ; \epsilon)=0 ; \frac{\partial \theta}{\partial \zeta}(\theta=1)=\frac{\partial \theta}{\partial \zeta}\left(\tau=\tau_{F R O N T}, \zeta=\zeta_{F R O N T} ; \epsilon\right)=0 \\
\theta \equiv \frac{\left(\bar{\rho}-\rho_{B O T}\right)}{\left(\rho_{T O P}-\rho_{B O T}\right)} ; \zeta \equiv z / d ; \tau \equiv K[\epsilon g / d]^{1 / 2} t ; \epsilon \equiv \Delta \rho / \rho_{B O T}
\end{gathered}
$$

Note that Eqs. (15)-(17) specify $\bar{\rho}(z=0)=\rho_{B O T}$. However, as discussed by Cannon and Zukoski $[1], \bar{\rho}$ at the base of the tube will always be somewhat greater than $\rho_{B O T}$, i.e.,

$\theta(\zeta=0 ; \tau>0)=\delta(\tau)$ where $\delta$ presumably satisfies $0 \ll \delta \ll 1$. Thus, the indicated boundary condition, $\theta(\zeta=0 ; \tau>0)=0$, of Eq. (16) is only approximately correct. In particular the solution to Eqs. (15)-(17) can be expected to provide a good simulation of experimental values only for moderate-to-large values of $\zeta$ and $\zeta_{\text {FRONT }}$.

Introducing the similarity variable $\eta$, it can be shown that 


$$
\theta=\theta(\eta ; \epsilon) ; \text { where } \eta=\zeta / \tau^{2 / 5}
$$

and that the problem of Eqs. (15)-(17) can be reduced to

$$
\begin{gathered}
\eta \frac{d \theta}{d \eta}=-\frac{5}{2} \frac{d}{d \eta}\left[\frac{1}{(1+\epsilon \theta)^{1 / 2}}\left(\frac{d \theta}{d \eta}\right)^{3 / 2}\right] ; 0 \leq \theta \leq 1 ; \\
\theta(\eta=0)=0 ; \frac{d \theta}{d \eta}(\theta=1)=\frac{d \theta}{d \eta}\left(\eta=\eta_{\text {FRONT }}\right)=0 ; \eta_{\text {FRONT }}=\eta_{\text {FRONT }}(\epsilon)
\end{gathered}
$$

where $\eta_{\text {FRONT }}$ is the smallest value of $\eta$ satisfying the above condition.

Once $\eta_{\text {PRONT }}(\epsilon)$ is obtained in the solution of Eqs. (19), the time-dependent position of the diffusion front can be determined from

$$
\zeta_{\text {FRONI }}(t, \epsilon)=z_{\text {FRONI }}(t, \epsilon) / d=\eta_{\text {FRONT }}(\epsilon)\left[K(\epsilon g / d)^{1 / 2} t\right]^{2 / 5}
$$

Solution for small $\epsilon$; verification with salt-water/fresh-water experiments. For the salt-water/freshwater experiments of Cannon and Zukoski [1], $\epsilon$ always satisfies $\epsilon<0.2$. Therefore, the $\epsilon=0$ solution te Eqs. (15)-(17) should provide a good simulation. This solution is found to be

$$
\begin{gathered}
\theta(\eta ; \epsilon=0)=\frac{3}{8}\left(\frac{\eta}{\eta_{F R O N T}}\right)\left[\left(\frac{\eta}{\eta_{F R O N I}}\right)^{4}-\frac{10}{3}\left(\frac{\eta}{\eta_{F R O N T}}\right)^{2}+5\right], 0 \leq \frac{\eta}{\eta_{F R O N T}} \leq 1 ; \\
\eta_{F R O N T}=\eta_{F R O N T}(\epsilon=0)=15^{3 / 5} / 8^{1 / 5}=3.3499 \ldots \\
\zeta_{\text {FRONT }}(t ; 0<\epsilon<1)=z_{\text {FRONT }}(t ; 0<\epsilon<<1) / d=\left(15^{3 / 5} / 8^{1 / 5}\right)\left[K(\epsilon g / d)^{1 / 2} t\right]^{2 / 5}
\end{gathered}
$$

Eq. (22) is plotted in Figure 3 for the $K^{\prime} s$ of Eqs. (13) and (14). (The $K=0.438$ plot will be explained below in the discussion of Phase II.)

The salt-water/fresh water experiments of Cannon and Zukoski [1] cover the $d$, L/d range $0.030 \mathrm{~m} \leq d \leq 0.181 \mathrm{~m}, 7.6 \leq L / d \leq 40.3$. For many, but not all of these experiments they tabulate (Table 6-2 of [1]) and/or plot (Figure 6.13 of [1]) $t_{\text {ARRVAL }}$. These data are included in Figure 3 . In 
the figure, note that when $K$ is taken to be $K_{G}=0.56$ the larger values of the $\zeta_{F R O N T}$ data are wellpredicted by Eq. (22). (Recall that this value of $K$ was also deduced from experiments in a saltwater/fresh-water system.) It is also noteworthy that $t_{A R R V A L}$ data for the largest $\nu_{d} d$ experiments $L / d=29.3$ and 40.3, are not reported in [1] and, therefore, are not included in Figure 3. However, data from these experiments up to $\zeta_{\text {FRONT }} \approx 25$ are reported. For times prior to $t_{A R R V A L}$, the $K=0.56$ plot of Figure 3 continues to predict well this data. Also, because of the aforementioned approximate nature of the $\eta=0$ boundary condition of Eqs. (19), and because the characteristic $d$ dimension of the turbulent-like eddies are associated with $\zeta$ intervals of the order of 1 , small-tomoderate values of $\zeta_{\text {FRONT }}$ are not expected to be well-simulated by the Eq. (22) solution.

Solution for moderate-to-large $\epsilon$; verification with heavy-gas/light-gas experiments. Simulations of the unsteady heavy-gas/light-gas experiments of Cannon and Zukoski [1] require solutions to Eqs. (19) with moderate-to-large values of $\epsilon$. To study such cases it is convenient to solve the following alternative coupled set of equations for $\theta, \psi$, and $\phi$,

$$
\begin{aligned}
& \qquad \begin{array}{c}
\frac{d \phi}{d \eta}=-\left(\frac{4}{15}\right) \eta \phi^{1 / 2}(1+\epsilon \theta)^{1 / 2}+\left(\frac{\epsilon}{3}\right) \frac{\phi^{2}}{(1+\epsilon \theta)} ; \\
\frac{d \psi}{d \eta}=\theta ; \frac{d \theta}{d \eta}=\phi, 0 \leq \theta \leq 1 \\
\text { where } \theta(\eta=0)=\psi(\eta=0)=0 \\
\text { and where } \Gamma(\epsilon) \equiv \phi(\eta=0)=\frac{d \theta}{d \eta}(\eta=0) \text { is determined such that } \\
\theta=1 ; \phi=\frac{d \theta}{d \eta}=0 \text { at some minimum } \eta \equiv \eta_{\text {FRONT }}(\epsilon)
\end{array}
\end{aligned}
$$

Solutions for

$$
\psi(\eta ; \epsilon)=\int_{0}^{\eta} \theta(\omega ; \epsilon) d \omega
$$

will be used below in the Phase II analysis.

Numerical integration of Eqs. (23) was carried out. This yielded $\theta(\eta ; \epsilon), \Gamma(\epsilon), \eta_{P R O N T}(\epsilon)$, and $\psi(\eta ; \epsilon)$ for $0<\epsilon \leq 40$. Some results are plotted in Figures 4a, 4b, and 4c, respectively. Figure 4a also includes a plot of the $\theta(\eta ; \epsilon=0)$ result of Eq. (21). As can be seen, although there is a significant variation in $\eta_{F R O N T}(\epsilon), \Gamma(\epsilon)$ and $\theta\left(\eta / \eta_{F R O N T} ; \epsilon\right)$ are relatively insensitive to differences in $\epsilon . \psi\left[\eta_{\text {FRONT }}(\epsilon) ; \epsilon\right]$ is plotted in Figure $4 \mathrm{~d}$. 
Cannon and Zukoski [1] report on Figure 2-type heavy-gas/light-gas experiments with $d=0.152 \mathrm{~m}$, $L / d=12$ and with $\epsilon=0.22,0.38$, and 4.0. Figure 6.14 of [1] presents Phase II data on $\theta\left(\zeta=L / d, \tau>\tau_{A R R V A L} ; \epsilon, L / d\right)$. However, Phase I data for these experiments are not provided. Also, note that estimates of $t_{A R R V A I}$ by extrapolation of the Phase II data to $\theta=1$ are subject to significant error because increases in $\epsilon$ lead to decreases in $t_{\text {ARRVVIL }}$ [e.g., $t_{A R R V V I}$ is estimated from Eq. (26) to be of the order of $5 \mathrm{~s}$ when $\epsilon=4.0$ ], and because of the "slow response time of the system used to measure the density of the gas mixtures [1]."

\section{Relaxation of the Diffusion Front: Phase II}

The initial value problem for Phase II. Subsequent to $t=t_{A R R V A L}$, during the relaxation of the diffusion front, $\theta$ is no longer characterized by the similarity variable. Rather, it must be determined from a solution of Eq. (15) subject to

$$
\begin{gathered}
\theta\left(\zeta=0, \tau>\tau_{A R R V A L} ; \epsilon\right)=\frac{\partial \theta}{\partial \zeta}\left(\zeta=L / d, \tau>\tau_{A R R V A L} ; \epsilon\right)=0 \\
\theta\left(\zeta, \tau=\tau_{A R R V A L} ; \epsilon\right) \equiv Z(\zeta ; \epsilon)=\theta\left(\eta=\zeta / \tau_{A R R V A L}^{2 / \zeta} ; \epsilon\right) \\
\tau_{A R R V A L} \equiv K[\epsilon g / d]^{1 / 2} t_{A R R V A L}=\left[(L / d) / \eta_{\text {FRONI }}(\epsilon)\right]^{5 / 2}
\end{gathered}
$$

An approximate solution. Partial verification of Eqs. (9)-(11) during Phase II can be obtained by an integral-type approximate solution to Eqs. (15) and (25). Toward this end, Eq. (15) is first integrated in $\zeta$ in the interval $0 \leq \zeta \leq L / d$. Using the boundary conditions of Eqs. (25), this leads to

$$
\frac{d}{d \tau}\left[\int_{0}^{L d} \theta(\zeta, \tau ; \epsilon) d \zeta\right]=-\left(\left.\frac{\partial \theta}{\partial \zeta}\right|_{\zeta=0}\right)^{3 / 2}
$$

Now choose the following approximate solution form, which satisfies exactly the initial/boundary conditions of Eq. (25).

$$
\theta=\theta\left(\zeta, \tau=\tau_{A R R V A I} ; \epsilon\right) T(\tau ; \epsilon, L / d) ; T\left(\tau=\tau_{A R R V A L} ; \epsilon, L / d\right)=1
$$

Using Eq. (28) in Eq. (27) eventually leads to 


$$
\begin{gathered}
T(\tau ; \epsilon, L / d)=\left\{\left[\Omega(\epsilon) /(L / d)^{5 / 2}\right]\left(\tau-\tau_{A R R N A D}\right)+1\right\}^{-2} \\
Q(\epsilon)=\left[\eta_{F R O N I}^{5 / 2}(\epsilon) \Gamma^{3 / 2}(\epsilon) / 2\right] / \psi\left[\eta_{\text {FRONI }}(\epsilon) ; \epsilon\right]
\end{gathered}
$$

where $Q(\epsilon)$ is plotted in Figure 5.

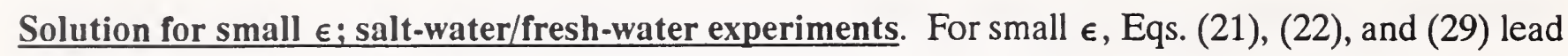
to

$$
\Omega(\epsilon=0)=\left(15^{3 / 2} / 2^{1 / 2}\right) / 11=1.8672 \ldots
$$

Using Eqs. (28)-(30), the Phase II solution at $z=L$ for $\epsilon \rightarrow 0$ and $L / d=15.3$ is plotted in Figure 6. This is the approximate solution that would be used to simulate Figure 2-type salt-water/freshwater experiments of Cannon and Zukoski [1]. The most accessible Phase II data from these experiments are presented in Figure 6.9 of [1] for the conditions: $d=0.089 m, L / d=15.3$, $\epsilon=0.050$. Reducing these data for plotting in Figure 6 requires the value of $K$. Two values were used, and corresponding reduced data sets were obtained and plotted. The first is $K=K_{G}=0.56$. The second is that value of $K$ which provides the least squares fit between the data and the theoretical solution curve. The latter value was found to be $K=0.438$, and, as can be seen in the figure, this provides an excellent match between the theory and the experiment during Phase II.

Using the "best-fit" $K=0.438$, a plot of the corresponding, theoretical, Phase I-solution has also been included in Figure 3. As can be seen, this value of $K$ and the corresponding solution provides a somewhat better match to the Phase I data than does that of the $K=0.56$ solution.

Solutions for moderate $\epsilon$; heavy-gas/light-gas experiments. Using Eqs. (28)-(29), the Phase II solutions at $z=L$ for $\epsilon=0.22,0.38$, and 4.0 are plotted in Figure 7. These approximate solutions are to be compared to the heavy-gas/light-gas data of Figure 6.14 of Cannon and Zukoski [1]. Again, reduction of the data for presentation in this figure requires a value of $K$. This is chosen to the previously determined "best-fit" value, $K=0.438$. As can be seen, the comparison between theory and experiment is good, especially for $\epsilon=0.22$ and 0.38 .

\section{SUMMARY, CONCLUSIONS, AND RECOMMENDATIONS}

A limitation of existing zone-type compartment fire models is the lack of a satisfactory means of simulating time-dependent fire-generated environments in ventilated shaft-like spaces with large height-to-span $(L / d)$ ratios. For such configurations, the traditional time-dependent, stably-stratified, spatially-uniform, two-layer description of a room fire environment, involving relatively-small-volume quasi-steady buoyant plumes is not valid. Rather, experimental observations previously reported in 
the literature suggest a time- and elevation-dependent density distribution, where any intervals of instability (i.e., density increasing with elevation) tend to be removed by the action of overturning and fluctuating turbulent-like mixing eddies of characteristic dimension, $d$. It has also been observed that on the macroscopic level, the mixing phenomenon has the features of a diffusion-like process.

Consistent with the above ideas and observations, a set of model equations to simulate combined buoyancy- and ventilation-driven flow of a perfect gas through a long vertical shaft was developed and presented as Eqs. (6)-(8), (10)-(11). Required to implement and solve this equation set is the value of a constant $K$. This is the coefficient of the equations' turbulent diffusivity term that leads to modifications in the density distribution in any regions along the length of the shaft where an unstable density distribution prevails.

The model equations were studied for a special class of problem involving no net ventilation flow velocity and no wall-to-gas heat transfer. Under such conditions all changes in density distribution are a direct result of the above diffusion-like mixing mechanism. Also, the equations are directly applicable to systems involving two-component fluids or gases.

For the above class of problem it was determined that with $K=0.44$, data from relevant, unsteady, Figure 2-type salt-water/fresh-water experiments of Cannon and Zukoski [1] are well-simulated by the equation set, Eqs. (9)-(11). For $L / d \geq 15$, excellent correlation between theory and experiment was found for both Phases I and II of the experiment. (Phase I is when the diffusion front, initiated at the bottom, moves up the shaft through the initially undisturbed liquid. Phase II begins when the front reaches the top of the closed shaft and continues until the unstable, top-to-bottom, density distribution relaxes asymptotically in time to a uniform state.) Data from additional Figure 2-type experiments involving heavy-gas/light-gas systems and relatively-large, initial, density differences $(\epsilon=0.22,0.38$, and 4.0 ) were also well-simulated by the Eq. (9)-(11), $K=0.44$ equation set.

The above results lead to some confidence in the validity of describing the diffusion-like mixing processes of interest by Eqs. (10) and (11) with $K=0.44$. In order to form an even greater level of confidence, additional comparisons between theory and experiment are necessary, especially in configurations different from that of Figure 2.

In view of the above it is recommended that the full equation set, Eqs. (6)-(8), (10)-(11), with $K=0.44$ be used in room fire models to describe the development of fire environments in ventilated, shaft-like, room configurations. This will require further development of the heat transfer elements of Eqs. (6) and (7), i.e., a verified method of simulating the $\dot{q}_{H T}^{\prime \prime \prime}$ term. The new large- $L / d$ model equations will be subject to verification with data from experiments on hot-air/cold-air systems and on fire-driven smoke flows, both of which involve gas-to-surface heat transfer exchanges. Such verification is required before the new shaft model can be used with confidence in professional practice. 


\section{REFERENCES}

[1] Cannon, J.B. and Zukoski, E.E, Turbulent Mixing in Vertical Shafts Under Conditions Applicable to Fires In High Rise Buildings, Tech. Report 1 of California Institute of Technology under NSF Grant GI31892X, Jan. 1976.

[2] Baird, M.H.I. and Rice, R.G., Axial Dispersion in Large Unbaffled Columns, Chemical Engineering, Vol. 9, pp. 171-174, 1975.

[3] Gardner, G.C., Motion of Miscible and Immiscible Fluids in Closed Horizontal and Vertical Ducts, International Journal of Multiphase Flow, Vol. 3, pp. 305-318, 1977.

[4] Mercer, A. and Thompson, H., An Experimental Investigation of Some Further Aspects of the Buoyancy-Driven Exchange Flow Between Carbon Dioxide and Air Following a Depressurization Accident in a Magnox Reactor, Journal of British Nuclear Energy Society, Vol. 14, pp. 327-340, 1975. 


\section{NOMENCLATURE}

$C_{p} \quad$ specific heat at constant pressure

$c_{B O T}, c_{\text {TOP }} \quad$ concentrations of top, bottom fluids

D eddy diffusivity

d characteristic span of a room or section of a shaft, e.g., diameter

$\boldsymbol{g} \quad$ acceleration of gravity

$\boldsymbol{K} \quad$ constant, Eq. (11)

$K_{G}, K_{B R} \quad$ Eqs. (13), (14)

$\boldsymbol{k} \quad$ thermal conductivity

L characteristic height of a compartment space, e.g., length of a shaft

$l \quad$ characteristic length of the fluid mixing process

$\dot{m}_{\text {VENT }}^{\prime \prime \prime} \quad$ rate of mass added to the shaft per unit volume

$Q \quad$ volume flow rate

$\dot{q}_{H T}^{\prime \prime \prime} \quad$ rate of heat transferred to the gas in the shaft per unit volume

$T \quad$ temperature in the shaft averaged across the section

$T_{V E N T} \quad T$ of gas flowing through a vent and into the shaft

$\bar{T}, T^{\prime} \quad$ time-averaged, fluctuating component of $T$

$t$ time

$t_{\text {ARRVAL }} \quad t$ when $z_{\text {FRONT }}=L$

$V_{z} \quad$ flow velocity in $\mathrm{z}$ direction average across a section

$\bar{V}_{z}, V_{z}^{\prime} \quad$ time-averaged, fluctuating component of $V_{z}$ 

$w^{\prime}$
characteristic velocity of the turbulent fluctuations
$z$ coordinate in upward direction
$z_{\text {FRONT }} \quad z$ of diffusion front
$\Gamma$
Eq. (23)
$\Delta \rho$
$\rho_{\text {TOP }}-\rho_{B O T}$
$\epsilon$
Eq. (17)
$\zeta$
Eq. (17)
$\zeta_{\text {FRONT }}$
$\zeta$ at the diffusion front
$\eta$
Eq. (18)
$\eta_{\text {FRONT }}$
$\eta$ at $\zeta=\zeta_{\text {FRONT }}$
$\theta$
Eq. (17)
$\rho$
density in the shaft averaged across the section
$\rho_{\text {AMB }}, \rho_{\text {VENT }} \rho$ of ambient, gas flowing through a vent and into the shaft
$\bar{\rho}, \rho^{\prime} \quad$ time-averaged, fluctuating component of $\rho$
$\rho_{A V E} \quad\left(\rho_{T O P}+\rho_{B O T}\right) / 2$
$\rho_{\text {TOP }}, \rho_{\text {BOT }} \rho$ of fluid in top, bottom of the vessels; initial $\rho$ at top, bottom of shaft
$\tau$
Eq. (17)
$\tau_{\text {ARRVAL }}$ Eq. (26)
$\phi \quad$ Eq. (23)
$\psi \quad$ Eqs. (23), (24)
$0 \quad$ Eq. (29)
$\omega \quad$ dummy variable, Eq. (24) 


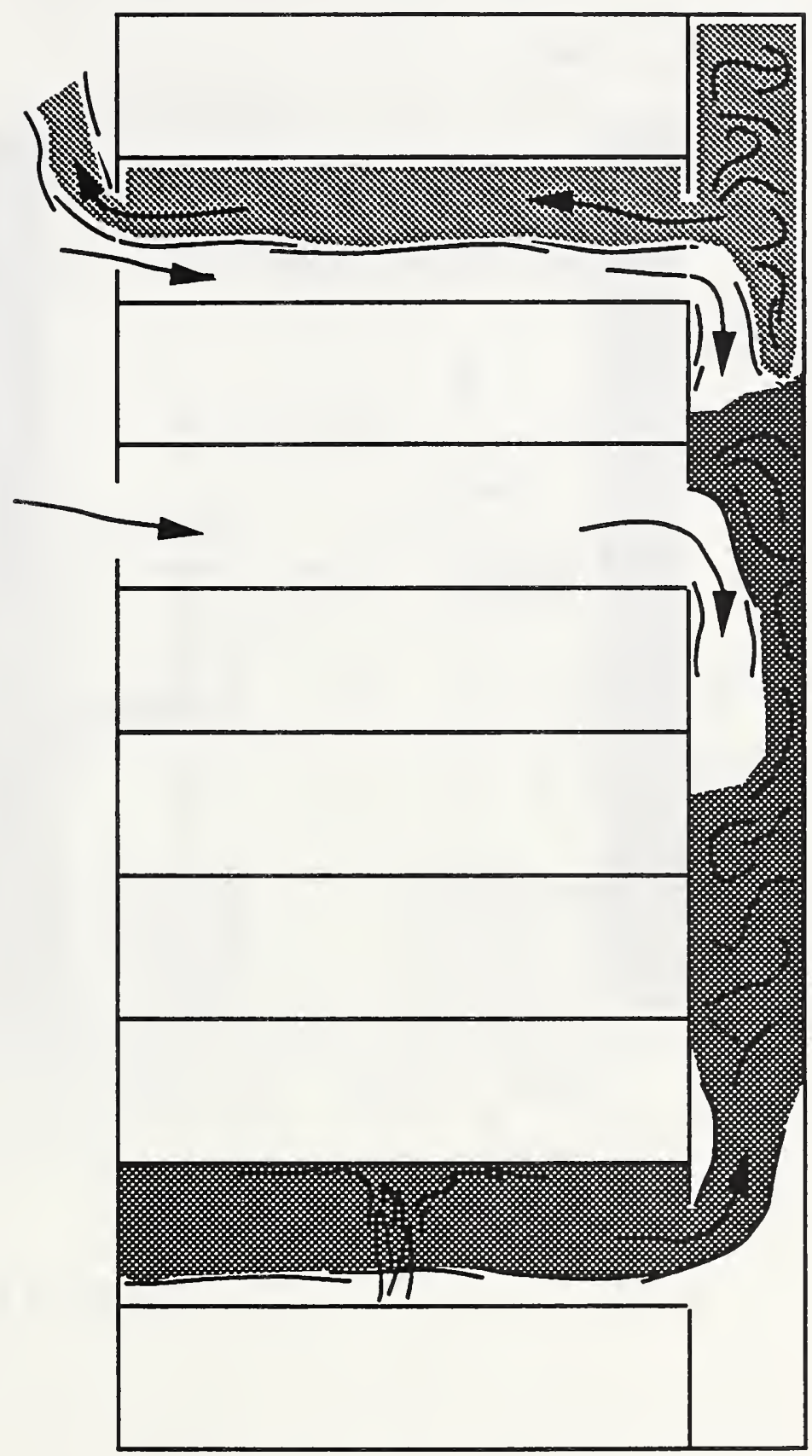

Figure 1. Sketch of a facility where traditional, two-layer, zone-type, modeling concepts are not uniformly applicable. 


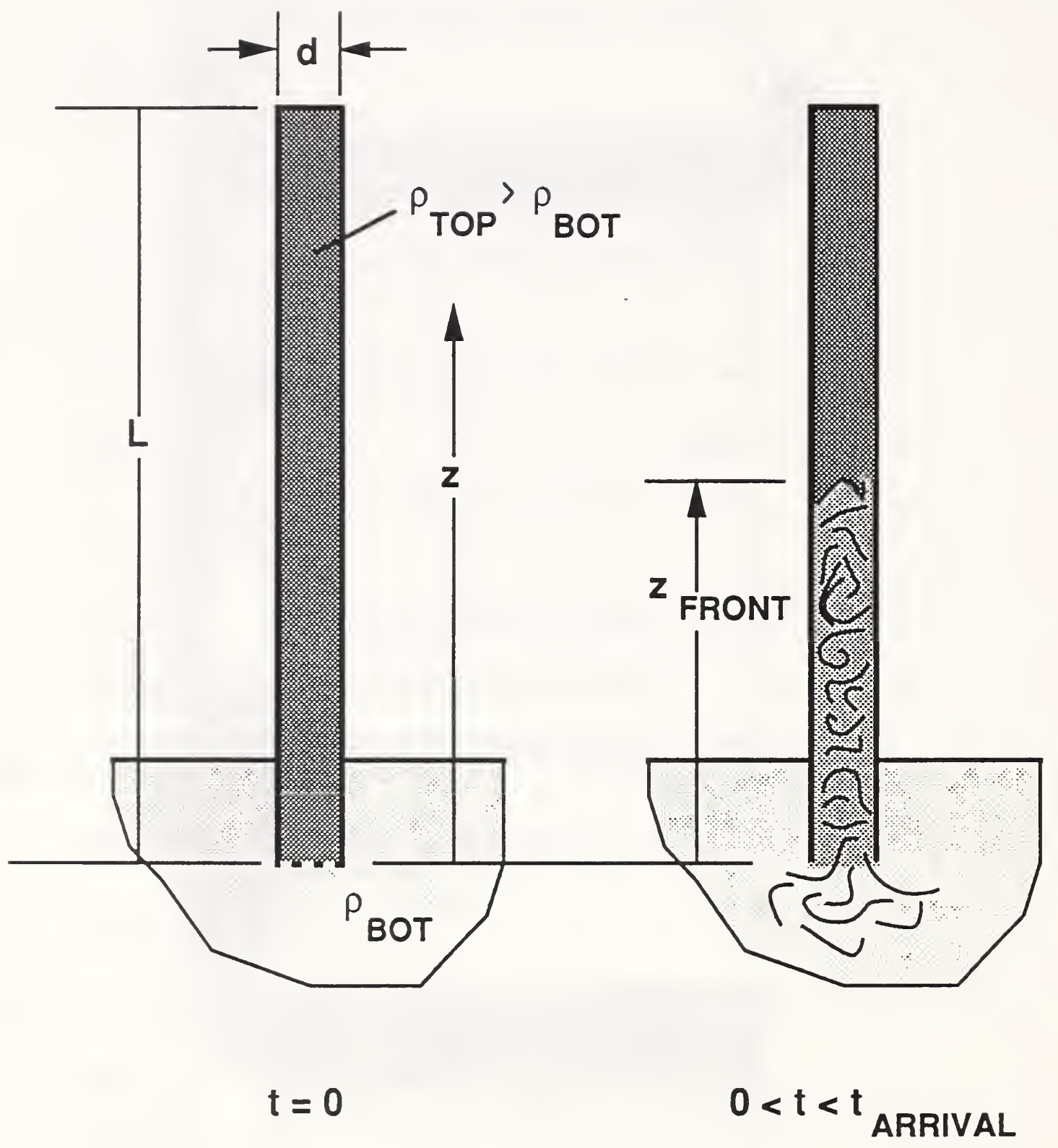

Figure 2. Sketch of the simulated experiments of Cannon and Zukoski [1]. 


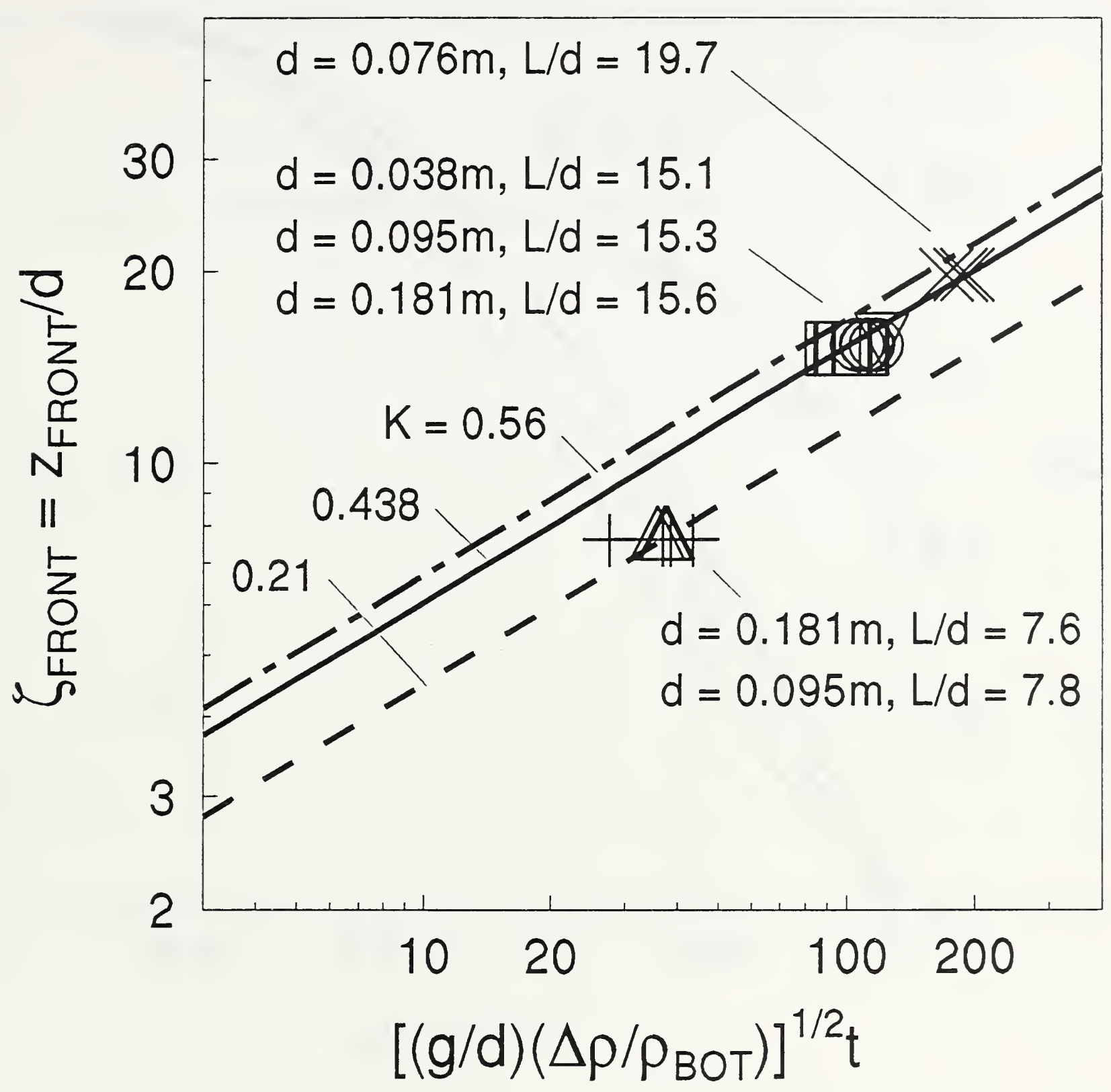

Figure 3. Position of the diffusion front in Phase I: small- $\epsilon$ solution of Eq. (22) for different values of $K$; and values measured in the salt-water/fresh-water experiments of Cannon and Zukoski [1]. 


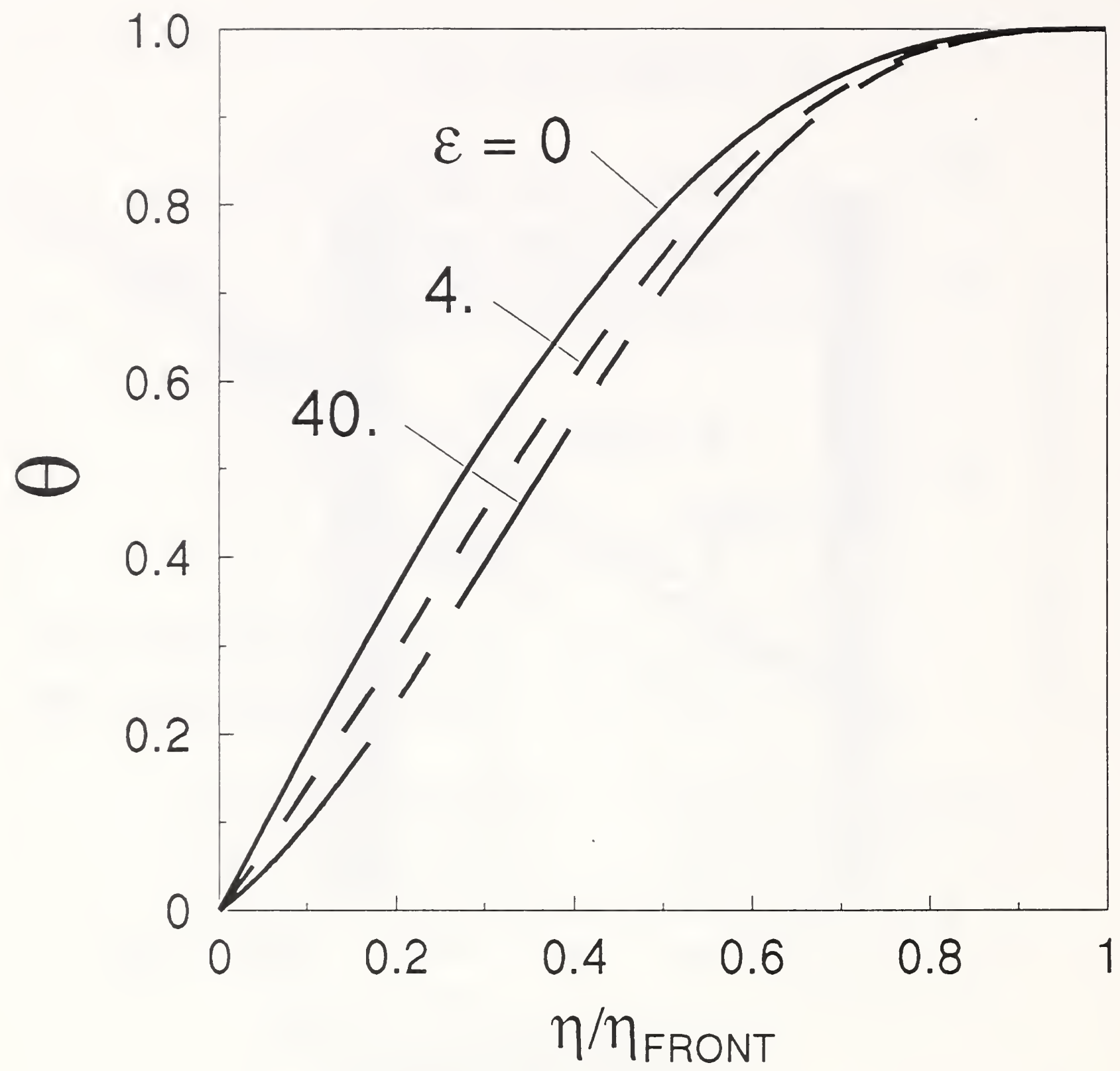

(a)

Figure 4. Phase I solutions of Eqs. (23), including moderate-to-large $\epsilon$ : (a) $\theta(\eta ; \epsilon)$; (b) $\Gamma(\epsilon)$; (c) $\eta_{F R O N T}(\epsilon)$; and (d) $\psi\left[\eta_{F R O N T}(\epsilon) ; \epsilon\right]$. 


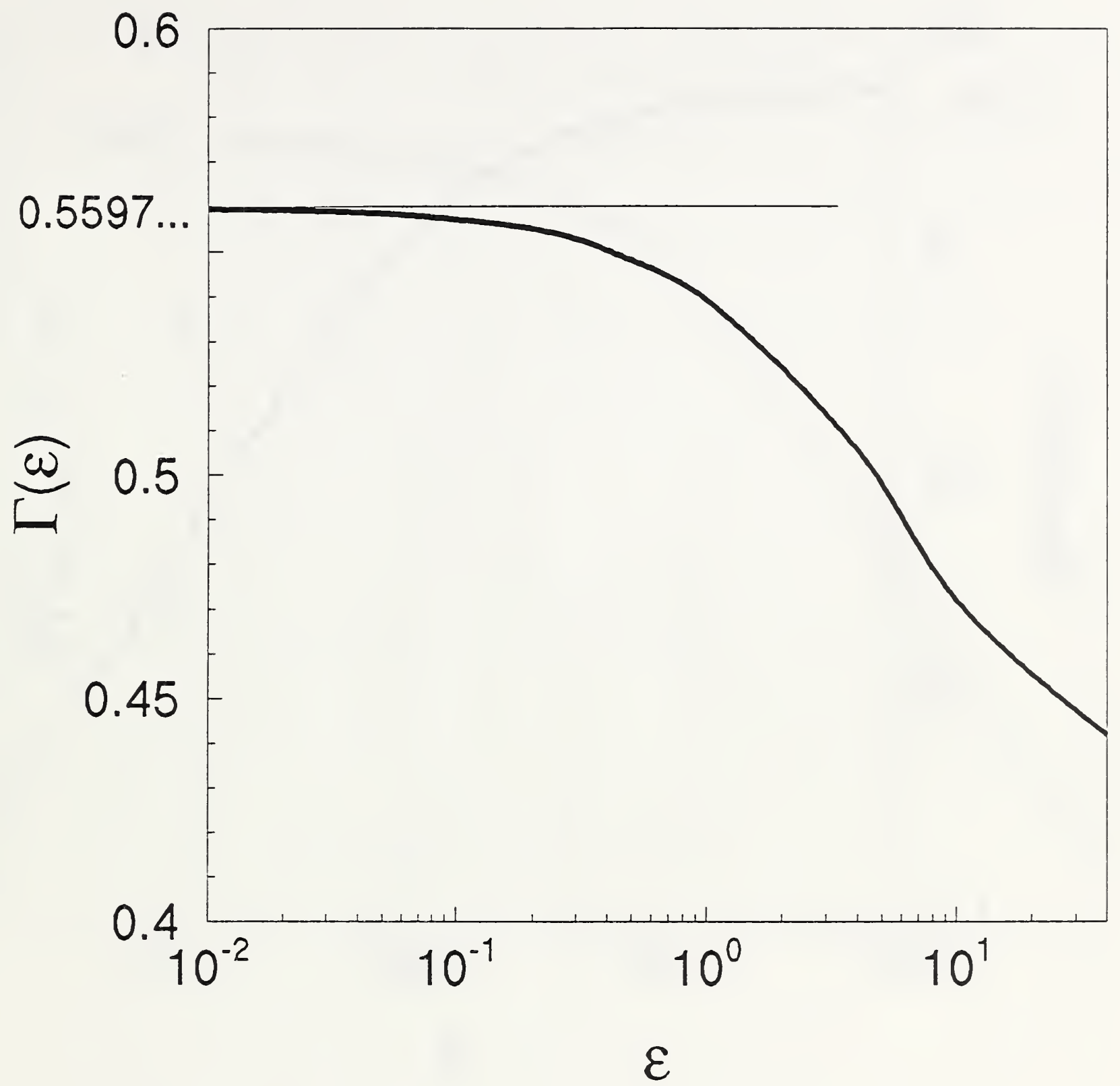

(b)

Figure 4. Phase I solutions of Eqs. (23), including moderate-to-large $\epsilon$ : (a) $\theta(\eta ; \epsilon)$;

(Cont'd) (b) $\Gamma(\epsilon)$; (c) $\eta_{F R O N T}(\epsilon)$; and (d) $\psi\left[\eta_{F R O N T}(\epsilon) ; \epsilon\right]$. 


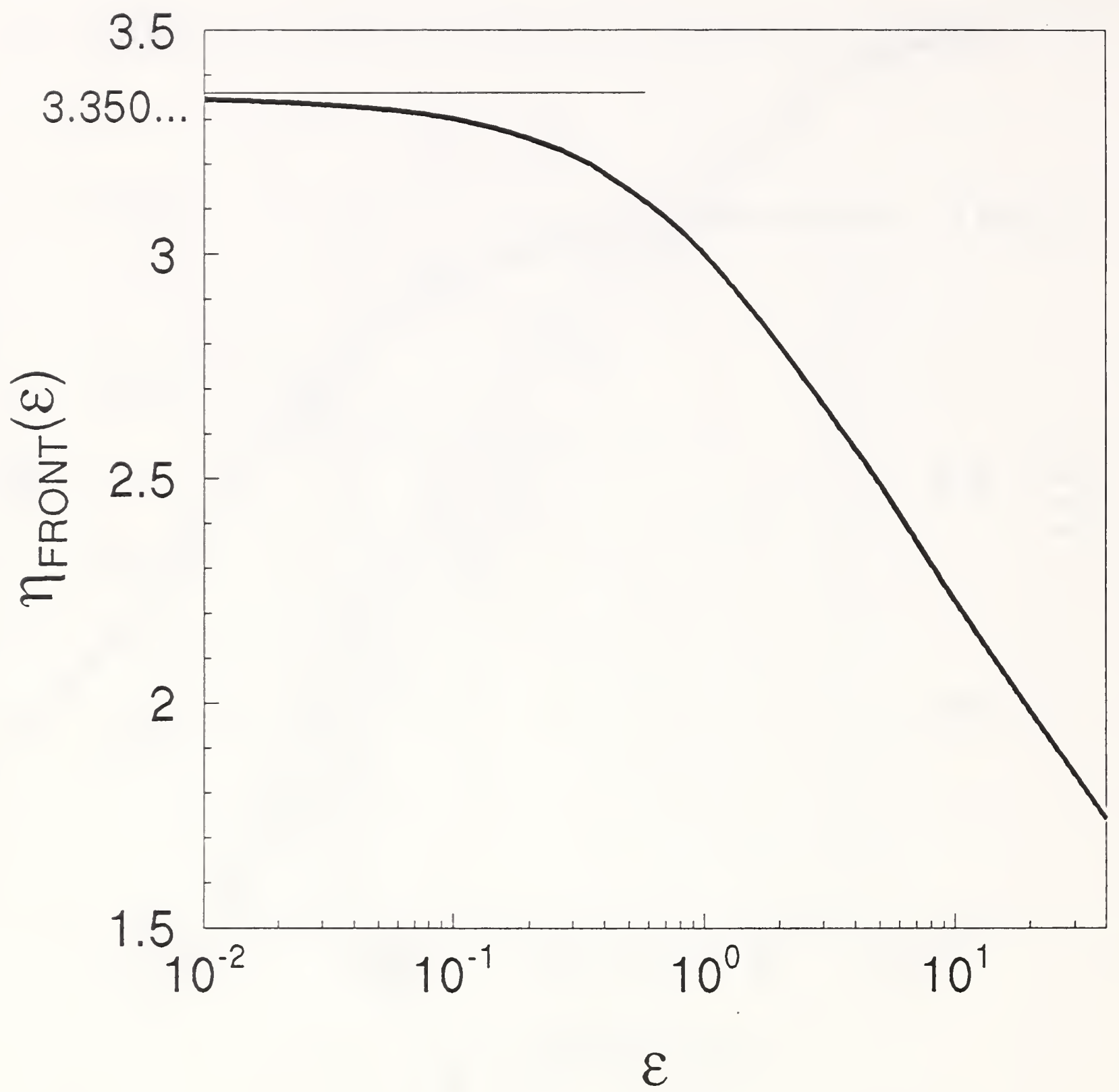

(c)

Figure 4. Phase I solutions of Eqs. (23), including moderate-to-large $\epsilon$ : (a) $\theta(\eta ; \epsilon)$; (Cont'd) $\quad$ (b) $\Gamma(\epsilon)$; (c) $\eta_{F R O N T}(\epsilon)$; and (d) $\psi\left[\eta_{F R O N T}(\epsilon) ; \epsilon\right]$ 


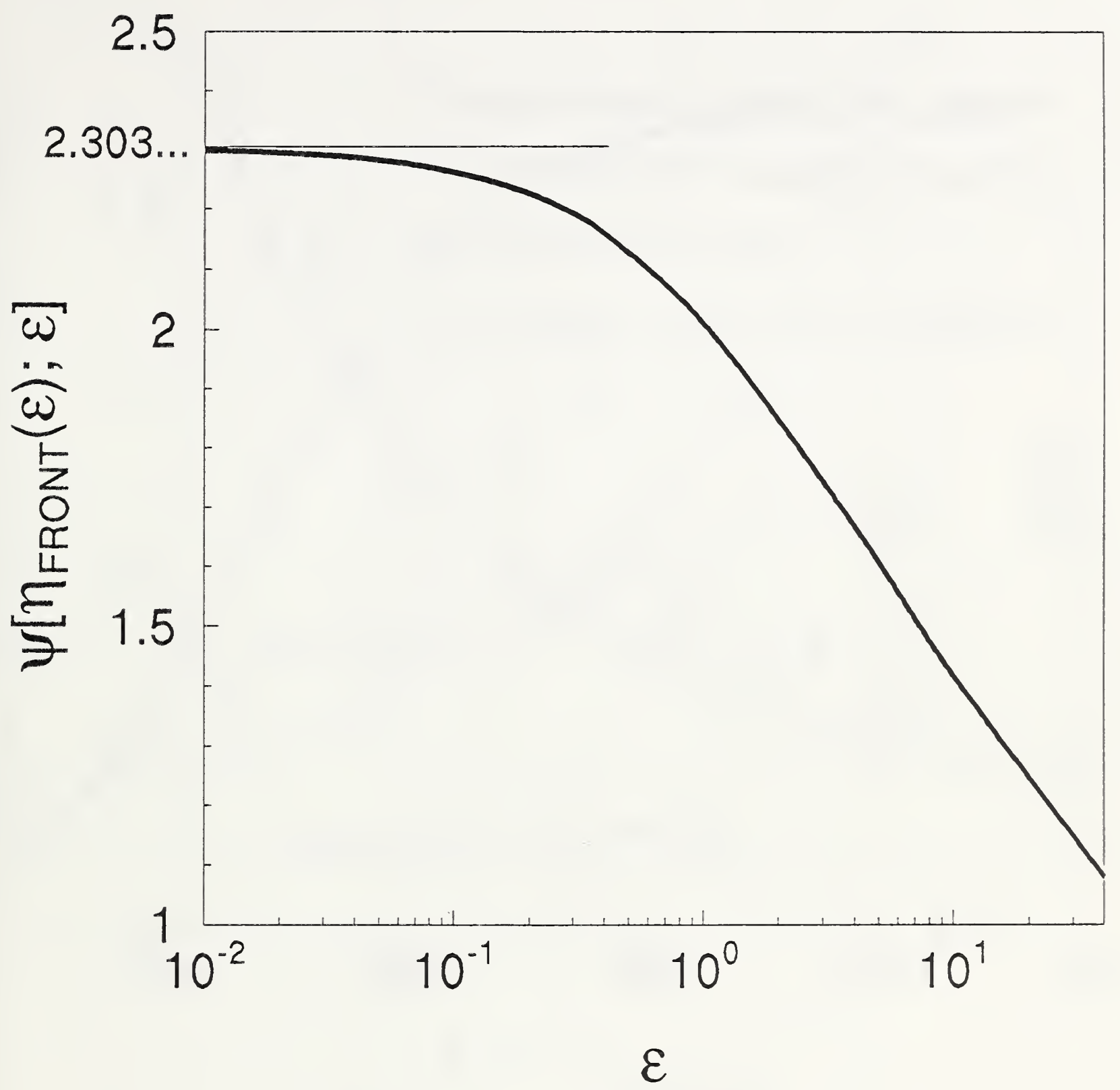

(d)

Figure 4. Phase I solutions of Eqs. (23), including moderate-to-large $\epsilon:$ (a) $\theta(\eta ; \epsilon)$;

(Cont'd) $\quad$ (b) $\Gamma(\epsilon) ;\left(\right.$ c) $\eta_{F R O N T}(\epsilon)$; and (d) $\psi\left[\eta_{F R O N T}(\epsilon) ; \epsilon\right]$. 


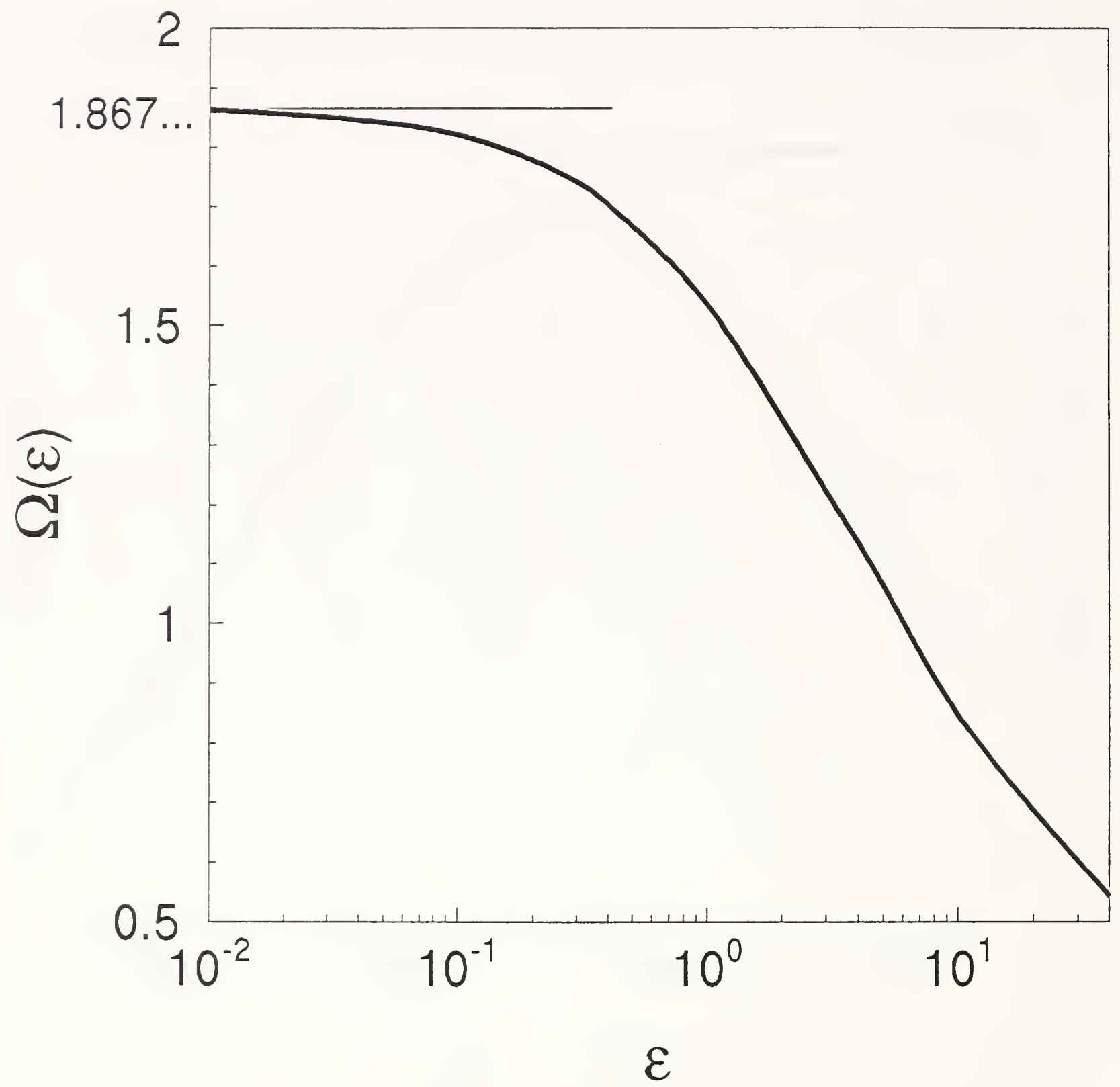

Figure 5. Plot of $Q(\epsilon)$ of Eq. (29) from Phase II solutions of Eqs. (27) and (28). 


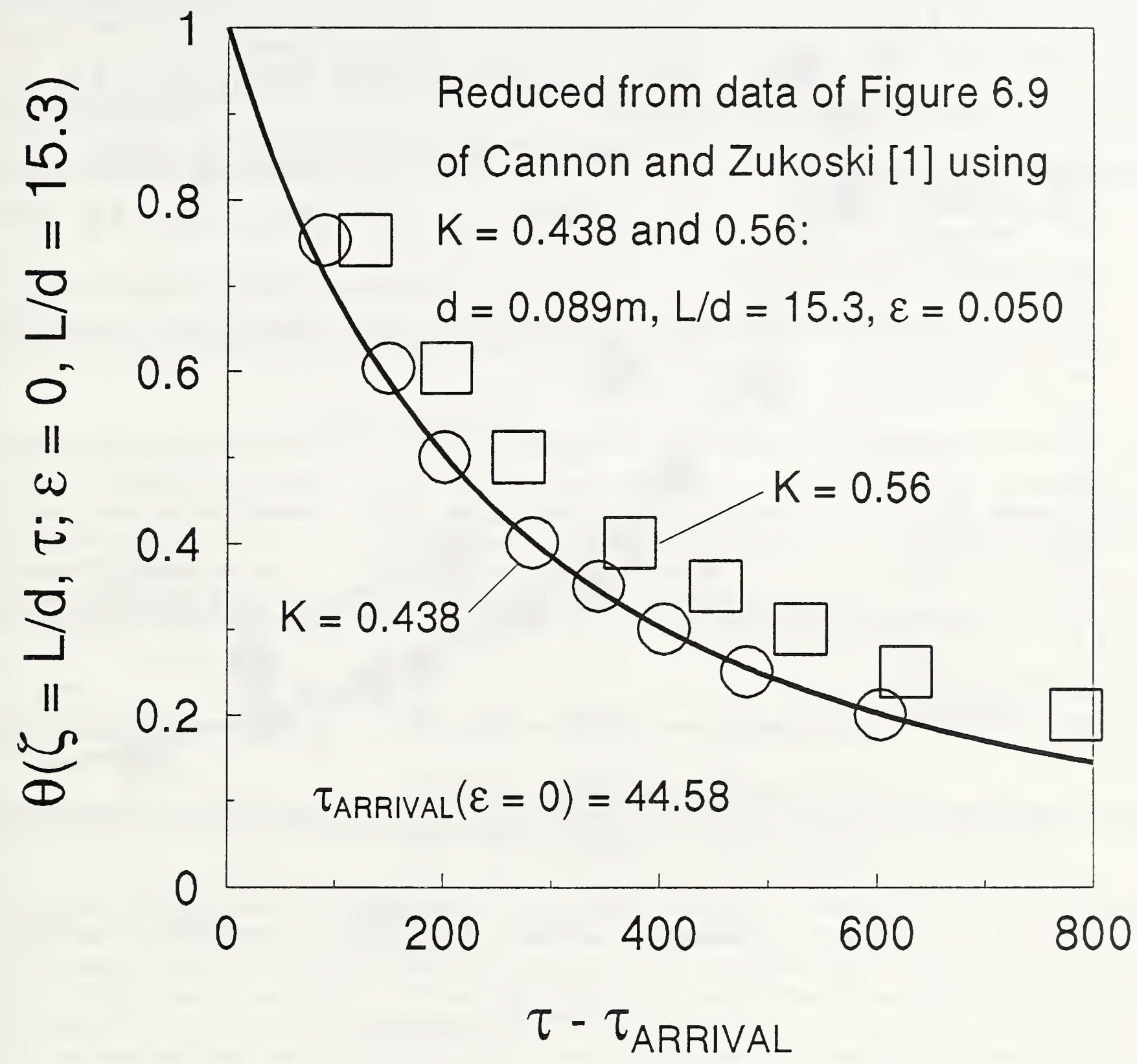

Figure 6. Plots for Phase II: Eqs. (28)-(29) at $z=L$ for $\epsilon=0$; salt-water/fresh-water data of Cannon and Zukoski [1] using $K=0.56$ and least-squares-fit value, $K=0.438$. 


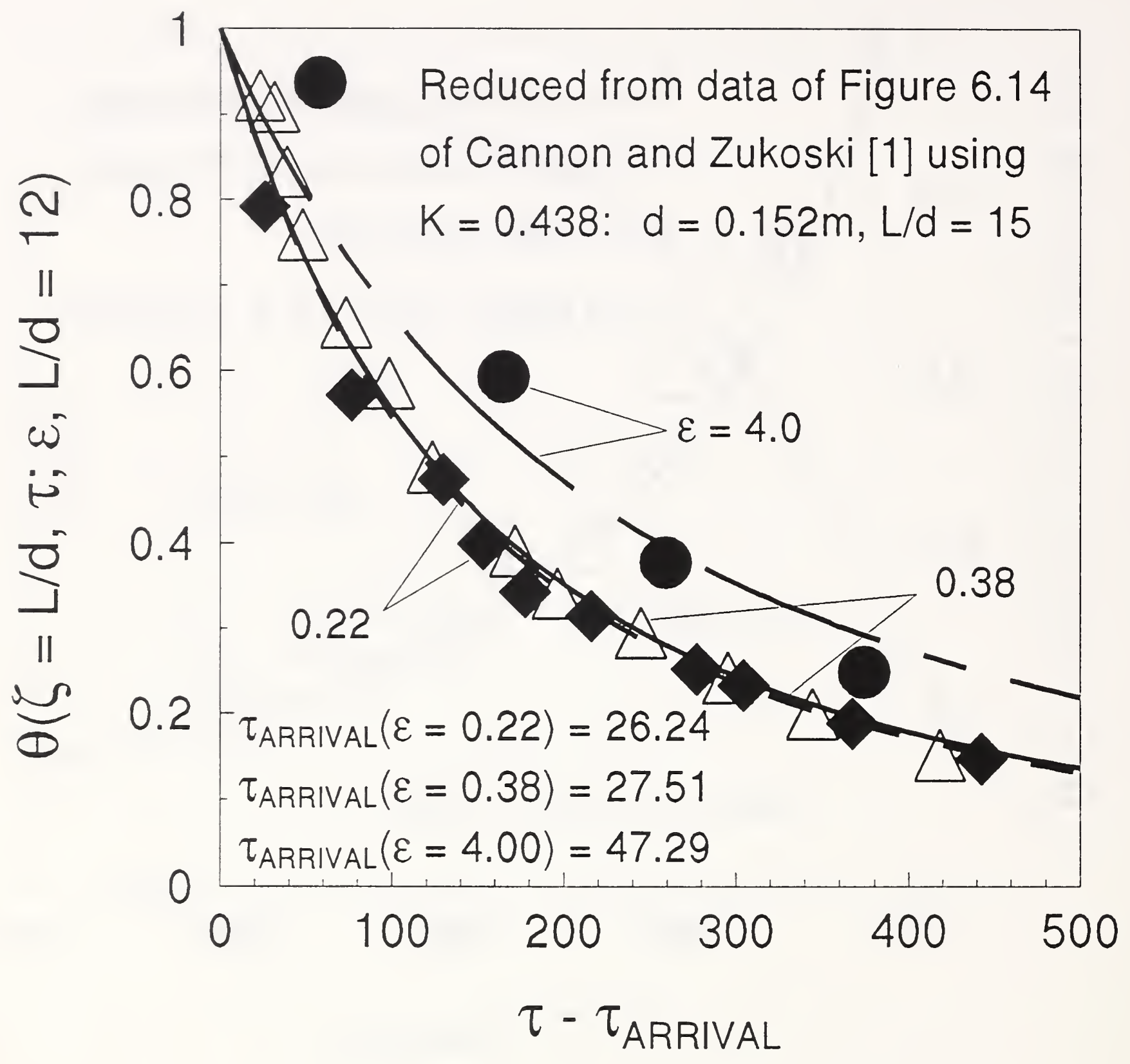

Figure 7. Plots for Phase II: Eqs. (28)-(29) at $z=L$ for $\epsilon=0.22,0.38$, and 4.0; heavy-gas/light-gas data of Cannon and Zukoski [1] for $\epsilon=0.22,0.38$, and 4.0 using $K=0.438$. 


